\title{
Lie symmetry analysis of some conformable fractional partial differential equations
}

Received: 19 April 2018 / Accepted: 29 November 2018 / Published online: 12 December 2018

(C) The Author(s) 2018

\begin{abstract}
In this article, Lie symmetry analysis is used to investigate invariance properties of some nonlinear fractional partial differential equations with conformable fractional time and space derivatives. The analysis is applied to Korteweg-de Vries, modified Korteweg-de Vries, Burgers, and modified Burgers equations with conformable fractional time and space derivatives. For each equation, all the vector fields and the Lie symmetries are obtained. Moreover, exact solutions are given to these equations in terms of solutions of ordinary differential equations. In particular, it is shown that the fractional Korteweg-de Vries can be reduced to the first Painlevé equation and to the fractional second Painlevé equation. In addition, a solution of the fractional modified Korteweg-de Vries is given in terms of solutions of the fractional second Painlevé equation.
\end{abstract}

Mathematics Subject Classification 26A33 $35 \mathrm{R} 11$

\section{Introduction}

In recent years, the interest in fractional calculus has increased due to its applications in many fields, such as Mathematics, Physics, Chemistry, Engineering, Finance, and Social sciences. As a result, several definitions for fractional derivatives appear in the literature to present more accurate models for real life phenomena. Some of known fractional derivatives are Riemann-Liouville, modified Riemann-Liouville, Caputo, Hadmard, Erdélyi-Kober, Riesz, Grünwald-Letnikov, Marchaud and others (see [11,12,26,27,29,31,33,34,37,40]). All known fractional derivatives satisfy one of the well-known properties of classical derivative, namely the linear property. However, the other properties of classical derivative, such as the derivative of a constant is zero; the product rule, quotient rule, and the chain rule, either do not hold or are too complicated for many fractional derivatives.

Recently, a new definition of fractional derivative that extends the familiar limit definition of the derivative of a function has been introduced by Khalil et al. [28]. The new definition is called the conformable fractional derivative. Unlike other definitions, this new definition is prominently compatible with the classical derivative and seems to satisfy all the requirements of the usual derivative. The importance of the conformable fractional derivative lies in satisfying the product and the quotient formulas. In [1], many properties of the conformable fractional derivative were proved, such as chain rule, exponential functions, Gronwall's inequality, integration by parts, Taylor power series expansions, and Laplace transforms. In [24], more results on the conformable fractional integral and derivative were given such as the extended mean value theorem and the Racetrack type principle. A conformable time-scale fractional calculus was introduced in [6] and a generalization of the conformable fractional derivative was given in [50]. Many studies related to this new fractional derivative were published $[2-4,15,19,32,41,43,51]$.

B. A. Tayyan · A. H. Sakka ( $\varangle)$

Department of Mathematics, Islamic University of Gaza, P. O. Box 108 Rimal, Gaza, Palestine

E-mail: asakka@mail.iugaza.edu.ps 
The Lie symmetry theory plays a significant role in the analysis of differential equations. The Norwegian mathematician Sophus Lie devoted the first work exclusively to the subject of Lie symmetry in the 19th century. It is regarded as the most important approach for constructing analytical solutions of nonlinear differential equations. After that, many papers and excellent textbooks have been devoted to the theory of Lie symmetry groups and their applications to differential equations; for examples, see [7-9,14,23,35]. Lie group analysis of fractional differential equations was investigated recently in $[5,10,13,16-18,20-22,25,30,36,38,39,44-$ 49]. The Lie symmetry analysis of time-fractional Burgers and Korteweg-de Vries (KdV) equations with Riemann-Liouville time derivative was studied in [39]. The Lie symmetry analysis of the KdV equations with modified Riemann-Liouville time-fractional derivative was investigated in [45]. It was shown that each of these equations can be reduced to a nonlinear ordinary differential equation of fractional order with a new independent variable. The fractional derivative in the reduced equations turned out to be the Erdelyi-Kober fractional derivative. In [42], the Lie symmetry analysis of Korteweg-de Vries, modified Korteweg-de Vries, Burgers, and modified Burgers equations with conformable fractional time derivative and classical space derivative has been investigated.

In this article, we derive the prolongation formulas for conformable fractional derivatives and apply the method of Lie group to conformable fractional partial differential equations (CFPDEs). We study the Lie symmetry analysis of Korteweg-de Vries, modified Korteweg-de Vries, Burgers, and modified Burgers equations with conformable fractional time and space partial derivatives. For each equation, all the vector fields and the Lie symmetries are obtained. We show that the equations under consideration can be reduced to ordinary differential equations with classical or fractional derivatives. In particular, we derive solutions of the conformable fractional Korteweg-de Vries and modified Korteweg-de Vries equations in terms of conformable fractional Painlevé equations.

\section{Conformable fractional calculus}

Definition 2.1 [28] Given a function $f:[0, \infty) \rightarrow \mathbb{R}$, the conformable fractional derivative of order $\alpha$ of $f$ is defined by

$$
D^{\alpha}[f(t)]=\lim _{\varepsilon \rightarrow 0} \frac{f\left(t+\varepsilon t^{1-\alpha}\right)-f(t)}{\varepsilon},
$$

for all $t>0, \alpha \in(0,1]$. If $D^{\alpha}[f(t)]$ exists for $t$ in some interval $(0, a), a>0$, and $\lim _{t \rightarrow 0^{+}} D^{\alpha}[f(t)]$ exists, then $D^{\alpha}[f(0)]=\lim _{t \rightarrow 0^{+}} D^{\alpha}[f(t)]$.

If $D^{\alpha}[f(t)]$ exists for $t \in[0, \infty)$, then $f$ is said to be $\alpha$-differentiable at $t$. One should notice that a function could be $\alpha$-differential at a point but not differentiable at the same point.

As an example $f(t)=\sqrt{t}, \quad D^{\frac{1}{2}}[f(t)]=\frac{1}{2}$. Consequently, $D^{\frac{1}{2}}[f(0)]=\frac{1}{2}$, but the first derivative is given by $D[f(0)]$, does not exist.

Theorem 2.2 [28] Let $\alpha \in(0,1]$ and $f, g$ be $\alpha$-differentiable at a point $t>0$. Then,

1. $D^{\alpha}[a f(t)+b g(t)]=a\left[D^{\alpha} f(t)\right]+b\left[D^{\alpha} g(t)\right]$, for all $a, b \in \mathbb{R}$.

2. If $f(t)=t^{p}$, then $D^{\alpha}[f(t)]=p t^{p-\alpha}$, for all $p \in \mathbb{R}$.

3. If $f$ is the constant function defined by $f(t)=c$, then $D^{\alpha}[f(t)]=0$.

4. $D^{\alpha}[f(t) g(t)]=f(t) D^{\alpha}[g(t)]+g(t) D^{\alpha}[f(t)]$.

5. $D^{\alpha}\left[\frac{f(t)}{g(t)}\right]=\frac{g(t) D^{\alpha}[f(t)]-f(t) D^{\alpha}[g(t)]}{[g(t)]^{2}}$.

6. If, in addition, $f$ is differentiable, then $D^{\alpha} f(t)=t^{1-\alpha} \frac{\mathrm{d} f(t)}{\mathrm{d} t}$.

Definition $2.3[28] I^{\alpha}[f(t)]=I\left[t^{\alpha-1} f(t)\right]=\int_{0}^{t} \frac{f(\tau)}{\tau^{1-\alpha}} d \tau$, where the integral is the usual Riemann improper integral, and $\alpha \in(0,1]$.

Theorem $2.4[28] D^{\alpha} I^{\alpha}[f(t)]=f(t)$, for $t \geq 0$, where $f$ is any continuous function in the domain of $I^{\alpha}$.

Lemma 2.5 [1] Let $f:[0, b) \rightarrow \mathbb{R}$ be differentiable and $0<\alpha \leq 1$. Then, for all $t>0$ we have $I^{\alpha} D^{\alpha}[f(t)]=f(t)-f(0)$.

Lemma 2.6 [1] Let $0<\alpha \leq 1, f$ be $\alpha$-differentiable at $g(t)>0$, and $g$ be $\alpha$-differentiable at $t>0$. Then, $D^{\alpha}[(f \circ g)(t)]=D^{\alpha}[f(g(t))] D^{\alpha}[g(t)][g(t)]^{\alpha-1}$. 
Lemma 2.7 [32] Let $0<\alpha \leq 1, f$ be differentiable at $g(t)$, and $g$ be $\alpha$-differentiable at $t>0$. Then, $D^{\alpha}[(f \circ g)(t)]=\left[f^{\prime}(g(t))\right] D^{\alpha}[g(t)]$.

\section{Lie symmetry analysis of CFPDEs}

Consider a conformable fractional partial differential equation in the form

$$
\frac{\partial^{\beta} u}{\partial t^{\beta}}=F\left(t, x, u, \frac{\partial^{\alpha} u}{\partial x^{\alpha}}, \frac{\partial^{2 \alpha} u}{\partial x^{2 \alpha}}, \frac{\partial^{3 \alpha} u}{\partial x^{3 \alpha}}, \cdots\right), \quad 0<\beta, \alpha \leq 1,
$$

where $u=u(x, t), F\left(t, x, u, \frac{\partial^{\alpha} u}{\partial x^{\alpha}}, \frac{\partial^{2 \alpha} u}{\partial x^{2 \alpha}}, \frac{\partial^{3 \alpha} u}{\partial x^{3 \alpha}}, \cdots\right)$ is a nonlinear function, $\frac{\partial^{\beta} u}{\partial t^{\beta}}$ and $\frac{\partial^{\alpha} u}{\partial x^{\alpha}}$ are the conformable fractional derivatives of order $\beta$ and $\alpha$, respectively. Here, $\frac{\partial^{n \alpha} u}{\partial x^{n \alpha}}$ are the sequential fractional derivatives given by

$$
\frac{\partial^{2 \alpha} u}{\partial x^{2 \alpha}}=\frac{\partial^{\alpha}}{\partial x^{\alpha}} \frac{\partial^{\alpha} u}{\partial x^{\alpha}}, \quad \frac{\partial^{n \alpha} u}{\partial x^{n \alpha}}=\frac{\partial^{\alpha}}{\partial x^{\alpha}} \frac{\partial^{(n-1) \alpha}}{\partial x^{(n-1) \alpha}}, \quad n=3,4, \cdots
$$

Our aim is to study the symmetry transformations of Eq. (2).

The invertible point transformations

$$
\hat{x}=X(t, x, u, \varepsilon), \quad \hat{t}=T(t, x, u, \varepsilon), \quad \hat{u}=U(t, x, u, \varepsilon),
$$

depending on a continuous parameter $\varepsilon$, are said to be symmetry transformations of Eq. (2), if Eq. (2) has the same form in the new variables $\hat{x}, \hat{t}, \hat{u}$. The set $G$ of all such transformations forms a continuous group. The symmetry group $G$ is also known as the group admitted by Eq. (2).

The key step in obtaining a Lie group of symmetry transformations is to find the infinitesimal generator of the group. To provide a basis of group generators, one has to create and solve the so-called determining system of equations.

The infinitesimal transformations of (3) read

$$
\begin{gathered}
\hat{x}=x+\varepsilon \xi(t, x, u)+o\left(\varepsilon^{2}\right), \\
\hat{t}=t+\varepsilon \tau(t, x, u)+o\left(\varepsilon^{2}\right), \\
\hat{u}=u+\varepsilon \eta(t, x, u)+o\left(\varepsilon^{2}\right) .
\end{gathered}
$$

It is convenient to introduce the operator

$$
V=\xi(t, x, u) \frac{\partial}{\partial x}+\tau(t, x, u) \frac{\partial}{\partial t}+\eta(t, x, u) \frac{\partial}{\partial u},
$$

which is known as the infinitesimal operator or the generator of the group $G$. The group transformations (3) corresponding to operator (5) can be obtained by solving the Lie equations

$$
\frac{d \hat{x}}{\mathrm{~d} \varepsilon}=\xi(\hat{t}, \hat{x}, \hat{u}), \quad \frac{d \hat{t}}{\mathrm{~d} \varepsilon}=\tau(\hat{t}, \hat{x}, \hat{u}), \quad \frac{d \hat{u}}{\mathrm{~d} \varepsilon}=\eta(\hat{t}, \hat{x}, \hat{u}),
$$

subject to the initial conditions

$$
\left.\hat{x}\right|_{\varepsilon=0}=x,\left.\quad \hat{t}\right|_{\varepsilon=0}=t,\left.\quad \hat{u}\right|_{\varepsilon=0}=u .
$$

A surface $u=u(t, x)$ is mapped to itself by the group of transformations generated by $V$ if

$$
V(u-u(t, x))=0 \quad \text { when } \quad u=u(t, x) .
$$

By definition, the transformations (3) form a symmetry group $G$ of Eq. (2) if the function $\hat{u}(\hat{t}, \hat{x})$ satisfies the equation

$$
\frac{\partial^{\beta} \hat{u}}{\partial \hat{t}^{\beta}}=F\left(\hat{t}, \hat{x}, \hat{u}, \frac{\partial^{\alpha} \hat{u}}{\partial \hat{x}^{\alpha}}, \frac{\partial^{2 \alpha} \hat{u}}{\partial \hat{x}^{2 \alpha}}, \frac{\partial^{3 \alpha} \hat{u}}{\partial \hat{x}^{3 \alpha}}, \cdots\right), \quad 0<\beta, \alpha \leq 1,
$$


whenever the function $u=u(t, x)$ satisfies Eq. (2). Extending transformation (4) to the operator of fractional differentiation $\frac{\partial^{\beta} u}{\partial t^{\beta}}$ and to the operator of fractional differentiation of various orders $\frac{\partial^{\alpha} u}{\partial x^{\alpha}}, \frac{\partial^{2 \alpha} u}{\partial x^{2 \alpha}}, \frac{\partial^{3 \alpha} u}{\partial x^{3 \alpha}}, \ldots$, one can obtain

$$
\begin{gathered}
\frac{\partial^{\beta} \hat{u}}{\partial \hat{t}^{\beta}}=\frac{\partial^{\beta} u}{\partial t^{\beta}}+\varepsilon \eta_{\beta}^{t}(t, x, u)+o\left(\varepsilon^{2}\right), \\
\frac{\partial^{\alpha} \hat{u}}{\partial \hat{x}^{\alpha}}=\frac{\partial^{\alpha} u}{\partial x^{\alpha}}+\varepsilon \eta_{\alpha}^{x}(t, x, u)+o\left(\varepsilon^{2}\right), \\
\frac{\partial^{2 \alpha} \hat{u}}{\partial \hat{x}^{2 \alpha}}=\frac{\partial^{2 \alpha} u}{\partial x^{2 \alpha}}+\varepsilon \eta_{\alpha}^{x x}(t, x, u)+o\left(\varepsilon^{2}\right), \\
\frac{\partial^{3 \alpha} \hat{u}}{\partial \hat{x}^{3 \alpha}}=\frac{\partial^{3 \alpha} u}{\partial x^{3 \alpha}}+\varepsilon \eta_{\alpha}^{x x x}(t, x, u)+o\left(\varepsilon^{2}\right), \\
\vdots
\end{gathered}
$$

where

$$
\begin{aligned}
\eta_{\beta}^{t}= & t^{1-\beta} \eta^{t}+(1-\beta) \tau t^{-\beta} u_{t}, \\
\eta_{\alpha}^{x}= & x^{1-\alpha} \eta^{x}+(1-\alpha) \xi x^{-\alpha} u_{x}, \\
\eta_{\alpha}^{x x}= & x^{2-2 \alpha} \eta^{x x}+(1-\alpha) x^{1-2 \alpha} \eta^{x}+(2-2 \alpha) x^{1-2 \alpha} \xi u_{x x} \\
& \quad+(1-\alpha)(1-2 \alpha) x^{-2 \alpha} \xi u_{x}, \\
\eta_{\alpha}^{x x x}= & x^{3-3 \alpha} \eta^{x x x}+(3-3 \alpha) x^{2-3 \alpha} \eta^{x x}+(1-\alpha)(1-2 \alpha) x^{1-3 \alpha} \eta^{x} \\
& +(3-3 \alpha) \xi x^{2-3 \alpha} u_{x x x}+(3-3 \alpha)(2-3 \alpha) \xi x^{1-3 \alpha} u_{x x} \\
& +(1-\alpha)(1-2 \alpha)(1-3 \alpha) \xi x^{-3 \alpha} u_{x},
\end{aligned}
$$

and

$$
\begin{aligned}
\eta^{t} & =D_{t}(\eta)-u_{x} D_{t}(\xi)-u_{t} D_{t}(\tau), \\
\eta^{x} & =D_{x}(\eta)-u_{x} D_{x}(\xi)-u_{t} D_{x}(\tau), \\
\eta^{x x} & =D_{x}\left(\eta^{x}\right)-u_{x x} D_{x}(\xi)-u_{x t} D_{x}(\tau), \\
\eta^{x x x} & =D_{x}\left(\eta^{x x}\right)-u_{x x x} D_{x}(\xi)-u_{x x t} D_{x}(\tau),
\end{aligned}
$$

Here, $D_{t}$ and $D_{x}$ denote the total derivative operators and are defined as:

$$
\begin{aligned}
& D_{t}=\frac{\partial}{\partial t}+u_{t} \frac{\partial}{\partial u}+u_{x t} \frac{\partial}{\partial u_{x}}+u_{t t} \frac{\partial}{\partial u_{t}}+\cdots \\
& D_{x}=\frac{\partial}{\partial x}+u_{x} \frac{\partial}{\partial u}+u_{x x} \frac{\partial}{\partial u_{x}}+u_{t x} \frac{\partial}{\partial u_{t}}+\cdots
\end{aligned}
$$

If the vector field (5) generates a symmetry of (2), then $V$ must satisfy Lie symmetry condition

$$
\left.\operatorname{pr}^{(n \alpha, \beta)} V\left(\Delta_{1}\right)\right|_{\Delta_{1}=0}=0,
$$

where $\Delta_{1}=\frac{\partial^{\beta} u}{\partial t^{\beta}}-F\left(t, x, u, \frac{\partial^{\alpha} u}{\partial x^{\alpha}}, \frac{\partial^{2 \alpha} u}{\partial x^{2 \alpha}}, \frac{\partial^{3 \alpha} u}{\partial x^{3 \alpha}}, \cdots\right)$. 


\section{The fractional Korteweg-de Vries equation}

In this section, we consider the following fractional Korteweg-de Vries (KdV) equation of the form

$$
\frac{\partial^{\beta} u}{\partial t^{\beta}}+6 u \frac{\partial^{\alpha} u}{\partial x^{\alpha}}+\frac{\partial^{3 \alpha} u}{\partial x^{3 \alpha}}=0,
$$

where $0<\beta, \alpha \leq 1, \beta$ and $\alpha$ are parameters describing the order of the conformable fractional time and space derivatives, respectively. According to the Lie theory, applying the $(3 \alpha, \beta)$ - prolongation $\operatorname{pr}^{(3 \alpha, \beta)} V$ to (14), we find the infinitesimal criterion (13) to be

$$
6 \eta \frac{\partial^{\alpha} u}{\partial x^{\alpha}}+\eta_{\beta}^{t}+6 u \eta_{\alpha}^{x}+\eta_{\alpha}^{x x x}=0
$$

which must be satisfied whenever $\frac{\partial^{\beta} u}{\partial t \beta}+6 u \frac{\partial^{\alpha} u}{\partial x^{\alpha}}+\frac{\partial^{3 \alpha} u}{\partial x^{3 \alpha}}=0$. It is worth to note that using Theorem (2.2), we find that (14) is equivalent to the following equation:

$$
\begin{aligned}
& t^{1-\beta} u_{t}+6 x^{1-\alpha} u u_{x}+(1-\alpha)(1-2 \alpha) x^{1-3 \alpha} u_{x} \\
& +3(1-\alpha) x^{2-3 \alpha} u_{x x}+x^{3-3 \alpha} u_{x x x}=0
\end{aligned}
$$

Substituting the general formulae for $\eta_{\alpha}^{x}, \eta_{\alpha}^{x x x}$ and $\eta_{\beta}^{t}$ from (11) and (12) into (15), using (16) to replace $u_{x x x}$ whenever it occurs, and equating the coefficients of the various monomials in partial derivatives of $u$, we can get the full determining equations for the symmetry group of (14). Solving these equations, we obtain

$$
\tau=\frac{-3 c_{1}}{2 \beta} t+c_{2} t^{1-\beta}, \quad \xi=\frac{-c_{1}}{2 \alpha} x+\frac{6 c_{3}}{\beta} t^{\beta} x^{1-\alpha}+c_{4} x^{1-\alpha}, \quad \eta=c_{1} u+c_{3}
$$

where $c_{1}, c_{2}, c_{3}$ and $c_{4}$ are arbitrary constants. Therefore, the symmetry group of (14) is spanned by the four vector fields

$$
\begin{array}{ll}
V_{1}=t^{1-\beta} \frac{\partial}{\partial t}, & V_{2}=x^{1-\alpha} \frac{\partial}{\partial x}, \\
V_{3}=\frac{6 t^{\beta} x^{1-\alpha}}{\beta} \frac{\partial}{\partial x}+\frac{\partial}{\partial u}, & V_{4}=\frac{-3 t}{2 \beta} \frac{\partial}{\partial t}-\frac{x}{2 \alpha} \frac{\partial}{\partial x}+u \frac{\partial}{\partial u} .
\end{array}
$$

The commutation relations between these vector fields are given by

$$
\begin{aligned}
& {\left[V_{1}, V_{2}\right]=0, \quad\left[V_{1}, V_{3}\right]=6 V_{2}, \quad\left[V_{1}, V_{4}\right]=\frac{-3}{2} V_{1},} \\
& {\left[V_{2}, V_{3}\right]=0, \quad\left[V_{2}, V_{4}\right]=\frac{-1}{2} V_{2}, \quad\left[V_{3}, V_{4}\right]=V_{3},}
\end{aligned}
$$

where the Lie bracket of two vector fields is defined by $[\rho, \sigma]=\rho \sigma-\sigma \rho$. Thus, we see that the set of these vector fields is closed under the Lie bracket.

The similarity variables for the infinitesimal generator $V_{4}$ can be found by solving the corresponding characteristic equation

$$
\frac{\alpha \mathrm{d} x}{x}=\frac{\beta \mathrm{d} t}{3 t}=\frac{-\mathrm{d} u}{2 u}
$$

and the corresponding invariants are

$$
\zeta=x t^{\frac{-\beta}{3 \alpha}}, \quad u=t^{\frac{-2 \beta}{3}} \Psi(\zeta)
$$

Substituting transformation (22) into (14), we find that (14) can be reduced to

$$
\frac{d^{3 \alpha} \Psi(\zeta)}{d \zeta^{3 \alpha}}+6 \Psi(\zeta) \frac{d^{\alpha} \Psi(\zeta)}{d \zeta^{\alpha}}-\frac{\beta}{3} \frac{\zeta^{\alpha}}{\alpha} \frac{d^{\alpha} \Psi(\zeta)}{d \zeta^{\alpha}}-\frac{2 \beta}{3} \Psi(\zeta)=0 .
$$


Equation (23) is a nonlinear fractional ordinary differential equation with conformable derivative. The scale $\omega=\left(\frac{\beta}{3}\right)^{\frac{1}{3 \alpha}} \zeta, \quad \Psi(\zeta)=\left(\frac{\beta}{3}\right)^{\frac{2}{3}} W(\omega)$ transforms (23) to an equivalent form

$$
K_{1}(W)=\frac{d^{3 \alpha} W(\omega)}{\mathrm{d} \omega^{3 \alpha}}+6 W(\omega) \frac{d^{\alpha} W(\omega)}{\mathrm{d} \omega^{\alpha}}-\frac{\omega^{\alpha}}{\alpha} \frac{d^{\alpha} W(\omega)}{\mathrm{d} \omega^{\alpha}}-2 W(\omega)=0
$$

Equation (24) can be integrated once using the following identity:

$$
\frac{d^{\alpha}}{d \omega^{\alpha}}\left[\left(2 W-\frac{\omega^{\alpha}}{\alpha}\right) K_{2}(W)\right]=\left(2 W-\frac{\omega^{\alpha}}{\alpha}\right) K_{1}(W)
$$

where

$$
K_{2}(W)=\frac{d^{2 \alpha} W}{\mathrm{~d} \omega^{2 \alpha}}+2 W^{2}-\frac{\omega^{\alpha}}{\alpha} W+\frac{\gamma(\gamma+1)+\frac{\mathrm{d}^{\alpha} W}{\mathrm{~d} \omega^{\alpha}}-\left(\frac{\mathrm{d}^{\alpha} W}{\mathrm{~d} \omega^{\alpha}}\right)^{2}}{2 W-\frac{\omega^{\alpha}}{\alpha}}=0 .
$$

Equation (26), under the transformation $\Theta=\left(W-\frac{\omega^{\alpha}}{2 \alpha}\right) /(4 \gamma+1)$, is reduced to the fractional thirty fourth Painlevé equation $\left(\mathrm{FP}_{34}\right)$

$$
\frac{d^{2 \alpha} \Theta}{\mathrm{d} \omega^{2 \alpha}}-\frac{1}{2 \Theta}\left(\frac{\mathrm{d}^{\alpha} \Theta}{d \omega^{\alpha}}\right)^{2}-4 \sigma \Theta^{2}+\frac{\omega^{\alpha}}{\alpha} \Theta+\frac{1}{2 \Theta}=0
$$

with $\sigma=\frac{-1}{6}$. The solutions of (26) are also expressible in terms of solutions of second fractional Painlevé equation $\left(F P_{I I}\right)$. There exists the following one-to-one correspondence between solutions of (26) and those of $F P_{I I}$, given by

$$
W=-\frac{\mathrm{d}^{\alpha} \Phi}{\mathrm{d} \omega^{\alpha}}-\Phi^{2}, \quad \Phi=\frac{\frac{\mathrm{d} \alpha W}{\mathrm{~d} \omega^{\alpha}}+\gamma}{2 W-\frac{\omega^{\alpha}}{\alpha}},
$$

where $\Phi$ satisfies the $F P_{I I}$ equation

$$
\frac{\mathrm{d}^{2 \alpha} \Phi(\omega)}{\mathrm{d} \omega^{2 \alpha}}=2 \Phi^{3}(\omega)+\frac{\omega^{\alpha}}{\alpha} \Phi(\omega)+\gamma
$$

As a second example, we consider the linear combination $V_{3}+a V_{1}$, where $a$ is a constant, to obtain another similarity reduction by solving the corresponding characteristic equation

$$
\frac{\beta \mathrm{d} x}{6 t^{\beta} x^{1-\alpha}}=\frac{\mathrm{d} t}{a t^{1-\beta}}=\frac{d u}{1}
$$

The corresponding invariants are

$$
\zeta=\frac{x^{\alpha}}{\alpha}-\frac{3}{a \beta^{2}} t^{2 \beta}, \quad u=\frac{1}{a \beta} t^{\beta}+\Psi(\zeta)
$$

Substituting transformation (31) into Eq. (14), one can find that (14) can be reduced to the following nonlinear ordinary differential equation with classical derivative:

$$
\frac{d^{3} \Psi(\zeta)}{\mathrm{d} \zeta^{3}}+6 \Psi(\zeta) \frac{\mathrm{d} \Psi(\zeta)}{\mathrm{d} \zeta}+\frac{1}{a}=0
$$

After a first integration, we get

$$
\frac{\mathrm{d}^{2} \Psi(\zeta)}{\mathrm{d} \zeta^{2}}+3 \Psi^{2}(\zeta)+\frac{1}{a} \zeta=\gamma
$$

where $\gamma$ is a constant of integration. Equation (33) is a second-order nonlinear differential equation with classical derivative and it can be reduced to the first Painlevé equation $\left(P_{I}\right)$

$$
\frac{d^{2} \Phi(z)}{\mathrm{d} z^{2}}=6 \Phi^{2}(z)+z
$$

via the change of variables $z=\left(\frac{1}{2 a}\right)^{\frac{1}{5}}(\zeta-\gamma a)$ and $\Psi(\zeta)=-2\left(\frac{1}{2 a}\right)^{\frac{2}{5}} \Phi(z)$. 


\section{The fractional modified Korteweg-de Vries equation}

This section investigates the Lie symmetry analysis of the fractional modified Korteweg-de Vries (mKdV) equation

$$
\frac{\partial^{\beta} u}{\partial t^{\beta}}-6 u^{2} \frac{\partial^{\alpha} u}{\partial x^{\alpha}}+\frac{\partial^{3 \alpha} u}{\partial x^{3 \alpha}}=0
$$

where $0<\beta, \alpha \leq 1$, and $\beta, \alpha$ are parameters describing the order of the conformable fractional time and space derivatives, respectively. According to the Lie theory, applying the $(3 \alpha, \beta)-$ prolongation $\mathrm{pr}^{(3 \alpha, \beta)} V$ to (35), we find the infinitesimal criterion (13) to be

$$
-12 \eta u \frac{\partial^{\alpha} u}{\partial x^{\alpha}}+\eta_{\beta}^{t}-6 \eta_{\alpha}^{x} u^{2}+\eta_{\alpha}^{x x x}=0,
$$

which must be satisfied whenever $\frac{\partial^{\beta} u}{\partial t^{\beta}}-6 u^{2} \frac{\partial^{\alpha} u}{\partial x^{\alpha}}+\frac{\partial^{3 \alpha} u}{\partial x^{3 \alpha}}=0$. Now, we write equation (35) in the equivalent form

$$
\begin{aligned}
& t^{1-\beta} u_{t}-6 x^{1-\alpha} u^{2} u_{x}+(1-\alpha)(1-2 \alpha) x^{1-3 \alpha} u_{x} \\
& +3(1-\alpha) x^{2-3 \alpha} u_{x x}+x^{3-3 \alpha} u_{x x x}=0 .
\end{aligned}
$$

Direct substitution of $\eta_{\alpha}^{x}, \eta_{\alpha}^{x x x}$ and $\eta_{\beta}^{t}$ from (11) and (12) into (36), using (37) to replace $u_{x x x}$ whenever it occurs, and equating the coefficients of the various monomials in partial derivatives of $u$, we get the full determining equations for the symmetry group of (35). Solving these equations, we obtain

$$
\tau=\frac{-3 c_{1}}{\beta} t+c_{2} t^{1-\beta}, \quad \xi=-\frac{c_{1}}{\alpha} x+c_{3} x^{1-\alpha}, \quad \eta=c_{1} u,
$$

where $c_{1}, c_{2}$ and $c_{3}$ are arbitrary constants. Therefore, the symmetry group of (35) is spanned by the three vector fields

$$
\begin{aligned}
& V_{1}=t^{1-\beta} \frac{\partial}{\partial t}, \quad V_{2}=x^{1-\alpha} \frac{\partial}{\partial x}, \\
& V_{3}=\frac{3 t}{\beta} \frac{\partial}{\partial t}+\frac{x}{\alpha} \frac{\partial}{\partial x}-u \frac{\partial}{\partial u} .
\end{aligned}
$$

These vector fields satisfy Lie bracket relations

$$
\left[V_{1}, V_{2}\right]=0, \quad\left[V_{1}, V_{3}\right]=3 V_{1}, \quad\left[V_{2}, V_{3}\right]=V_{2} .
$$

Note that when $\beta=\alpha=1$, the vector fields of the fractional $\mathrm{mKdV}$ equation reduce to the vector fields of the classical $\mathrm{mKdV}$ equation [7].

The similarity variables for the infinitesimal generator $V_{3}$ can be found by solving the corresponding characteristic equations

$$
\frac{\alpha \mathrm{d} x}{x}=\frac{\beta \mathrm{d} t}{3 t}=\frac{\mathrm{d} u}{-u}
$$

The corresponding invariants are

$$
\zeta=x t^{\frac{-\beta}{3 \alpha}}, \quad u=t^{\frac{-\beta}{3}} \Psi(\zeta) .
$$

Using the transformation (42), Eq. (35) can be reduced to the nonlinear FODE

$$
\frac{d^{3 \alpha} \Psi(\zeta)}{\mathrm{d} \zeta^{3 \alpha}}-6 \Psi^{2}(\zeta) \frac{d^{\alpha} \Psi(\zeta)}{\mathrm{d} \zeta^{\alpha}}-\frac{\beta}{3} \frac{\zeta^{\alpha}}{\alpha} \frac{d^{\alpha} \Psi(\zeta)}{\mathrm{d} \zeta^{\alpha}}-\frac{\beta}{3} \Psi(\zeta)=0 .
$$

As a result, we have

$$
\frac{d^{2 \alpha} \Psi(\zeta)}{\mathrm{d} \zeta^{2 \alpha}}-2 \Psi^{3}(\zeta)-\frac{\beta}{3} \frac{\zeta^{\alpha}}{\alpha} \Psi(\zeta)=\gamma
$$


where $\gamma$ is a constant of integration. Equation (44) can be converted by the scale $\omega=\left(\frac{\beta}{3}\right)^{\frac{1}{3 \alpha}} \zeta, \Psi(\zeta)=$ $\left(\frac{\beta}{3}\right)^{\frac{1}{3}} \Phi(\omega)$ to the fractional Painlevé equation $F P_{I I}$

$$
\frac{d^{2 \alpha} \Phi(\omega)}{\mathrm{d} \omega^{2 \alpha}}-2 \Phi^{3}(\omega)-\frac{\omega^{\alpha}}{\alpha} \Phi(\omega)=\mu
$$

where $\mu=3 \gamma$.

\section{The fractional Burgers equation}

This section is devoted to the Lie symmetry analysis of the following fractional Burgers equation

$$
\frac{\partial^{\beta} u}{\partial t^{\beta}}+a u \frac{\partial^{\alpha} u}{\partial x^{\alpha}}+b \frac{\partial^{2 \alpha} u}{\partial x^{2 \alpha}}=0
$$

where $0<\beta, \alpha \leq 1, \beta$ and $\alpha$ are parameters describing the order of the conformable fractional time and space derivatives. According to the Lie theory, applying the $(2 \alpha, \beta)$-prolongation $\operatorname{pr}^{(2 \alpha, \beta)} V$ to (46), the infinitesimal criterion (13) reads

$$
a \eta \frac{\partial^{\alpha} u}{\partial x^{\alpha}}+\eta_{\beta}^{t}+a u \eta_{\alpha}^{x}+b \eta_{\alpha}^{x x}=0 .
$$

The condition (47) must be satisfied whenever $\frac{\partial^{\beta} u}{\partial t^{\beta}}+a u \frac{\partial^{\alpha} u}{\partial x^{\alpha}}+b \frac{\partial^{2 \alpha} u}{\partial x^{2 \alpha}}=0$. Equation (46) has the equivalent form

$$
t^{1-\beta} u_{t}+a x^{1-\alpha} u u_{x}+b(1-\alpha) x^{1-2 \alpha} u_{x}+b x^{2-2 \alpha} u_{x x}=0 .
$$

Next, we use (11) and (12) to substitute $\eta_{\alpha}^{x}, \eta_{\alpha}^{x x}$ and $\eta_{\beta}^{t}$ into (47), and (48) to replace $u_{x x}$ whenever it occurs. After equating the coefficients of the various monomials in partial derivatives of $u$, we get the full determining equations for the symmetry group of (46). Solving these equations, we obtain

$$
\begin{aligned}
& \tau=\frac{c_{1} t^{1+\beta}}{\beta^{2}}-\frac{2 c_{2} t}{\beta}+c_{4} t^{1-\beta}, \\
& \xi=\frac{c_{1} x t^{\beta}}{\alpha \beta}-\frac{c_{2} x}{\alpha}+\frac{a c_{3}}{\beta} t^{\beta} x^{1-\alpha}+c_{5} x^{1-\alpha}, \\
& \eta=\left(\frac{-c_{1} t^{\beta}}{\beta}+c_{2}\right) u+\left(\frac{c_{1} x^{\alpha}}{a \alpha}+c_{3}\right),
\end{aligned}
$$

where $c_{1}, c_{2}, c_{3}, c_{4}$ and $c_{5}$ are arbitrary constants. Therefore, the symmetry group of (46) is spanned by the five vector fields

$$
\begin{aligned}
& V_{1}=t^{1-\beta} \frac{\partial}{\partial t}, \quad V_{2}=x^{1-\alpha} \frac{\partial}{\partial x} \\
& V_{3}=\frac{a t^{\beta} x^{1-\alpha}}{\beta} \frac{\partial}{\partial x}+\frac{\partial}{\partial u}, \quad V_{4}=\frac{-2 t}{\beta} \frac{\partial}{\partial t}-\frac{x}{\alpha} \frac{\partial}{\partial x}+u \frac{\partial}{\partial u}, \\
& V_{5}=\frac{t^{1+\beta}}{\beta^{2}} \frac{\partial}{\partial t}+\frac{x t^{\beta}}{\alpha \beta} \frac{\partial}{\partial x}+\left(\frac{-t^{\beta} u}{\beta}+\frac{x^{\alpha}}{a \alpha}\right) \frac{\partial}{\partial u} .
\end{aligned}
$$

It is easily checked that these five vector fields satisfy

$$
\begin{aligned}
& {\left[V_{1}, V_{2}\right]=\left[V_{2}, V_{3}\right]=\left[V_{3}, V_{5}\right]=0, \quad\left[V_{2}, V_{4}\right]=-V_{2},} \\
& {\left[V_{2}, V_{5}\right]=\frac{1}{a} V_{3},\left[V_{1}, V_{3}\right]=a V_{2}, \quad\left[V_{1}, V_{4}\right]=-2 V_{1},} \\
& {\left[V_{1}, V_{5}\right]=-V_{4}, \quad\left[V_{3}, V_{4}\right]=V_{3}, \quad\left[V_{4}, V_{5}\right]=-2 V_{5} .}
\end{aligned}
$$


Thus, the Lie algebra of infinitesimal symmetries of equation (46) is spanned by these five vector fields. The number of the vector fields coincides with that of the classical Burgers equation and when $\beta=\alpha=1$ these vector fields reduce to that of the classical Burgers equation [14].

The similarity variables for the infinitesimal generator $V_{4}$ can be found by solving the corresponding characteristic equation

$$
\frac{-\alpha \mathrm{d} x}{x}=\frac{\beta \mathrm{d} t}{-2 t}=\frac{\mathrm{d} u}{u}
$$

and the corresponding invariants are

$$
\zeta=x t^{\frac{-\beta}{2 \alpha}}, \quad u=t^{\frac{-\beta}{2}} \Psi(\zeta)
$$

The transformation (53) reduces Eq. (46) to the following nonlinear $F O D E$

$$
b \frac{d^{2 \alpha} \Psi(\zeta)}{d \zeta^{2 \alpha}}+a \Psi(\zeta) \frac{d^{\alpha} \Psi(\zeta)}{\mathrm{d} \zeta^{\alpha}}-\frac{\beta}{2} \frac{\zeta^{\alpha}}{\alpha} \frac{d^{\alpha} \Psi(\zeta)}{\mathrm{d} \zeta^{\alpha}}-\frac{\beta}{2} \Psi(\zeta)=0 .
$$

Consequently, we have

$$
b \frac{d^{\alpha} \Psi(\zeta)}{\mathrm{d} \zeta^{\alpha}}+\frac{a}{2} \Psi^{2}(\zeta)-\frac{\beta}{2} \frac{\zeta^{\alpha}}{\alpha} \Psi(\zeta)=\gamma,
$$

where $\gamma$ is a constant of integration.

The fractional Riccati equation (55) can be transformed by the transform $\Psi(\zeta)=\frac{2 b}{a} \Phi^{-1}(\zeta) \frac{d^{\alpha} \Phi(\zeta)}{d \zeta^{\alpha}}$ to the linear equation

$$
\frac{d^{2 \alpha} \Phi(\zeta)}{\mathrm{d} \zeta^{2 \alpha}}-\frac{\beta}{2 b} \frac{\zeta^{\alpha}}{\alpha} \frac{d^{\alpha} \Phi(\zeta)}{\mathrm{d} \zeta^{\alpha}}+\frac{\gamma}{b} \Phi(\zeta)=0
$$

From the linear combination $V_{3}+\mu V_{1}$, where $\mu$ is a constant, another similarity reduction can be found by solving the corresponding characteristic equation

$$
\frac{\beta \mathrm{d} x}{a t^{\beta} x^{1-\alpha}}=\frac{\mathrm{d} t}{\mu t^{1-\beta}}=\frac{\mathrm{d} u}{1}
$$

and the corresponding invariants are

$$
\zeta=\frac{x^{\alpha}}{\alpha}-\frac{a}{2 \mu \beta^{2}} t^{2 \beta}, \quad u=\frac{1}{\mu \beta} t^{\beta}+\Psi(\zeta) .
$$

Substituting transformation (58) into Eq. (46), we find that (46) can be reduced to a nonlinear $O D E$ with the classical derivative

$$
b \Psi^{\prime \prime}(\zeta)+a \Psi(\zeta) \Psi^{\prime}(\zeta)+\frac{1}{\mu}=0,
$$

where $\Psi^{\prime}(\zeta):=\frac{d \Psi(\zeta)}{d \zeta}$. From which we obtain the Riccati equation

$$
b \Psi^{\prime}(\zeta)+\frac{a}{2} \Psi^{2}(\zeta)+\frac{1}{\mu} \zeta=\gamma,
$$

where $\gamma$ is a constant of integration. 


\section{The fractional modified Burgers equation}

In this section, we will consider the Lie symmetry analysis of the following nonlinear fractional modified Burgers equation

$$
\frac{\partial^{\beta} u}{\partial t^{\beta}}+a u^{2} \frac{\partial^{\alpha} u}{\partial x^{\alpha}}+b \frac{\partial^{2 \alpha} u}{\partial x^{2 \alpha}}=0
$$

where $0<\beta, \alpha \leq 1, \beta$ and $\alpha$ are parameters describing the order of the conformable fractional time and space derivatives. According to the Lie theory, applying the $(2 \alpha, \beta)$-prolongation $\operatorname{pr}^{(2 \alpha, \beta)} V$ to (61), one can find the infinitesimal criterion (13) to be

$$
2 a \eta x^{1-\alpha} u u_{x}+\eta_{\beta}^{t}+a \eta_{\alpha}^{x} u^{2}+b \eta_{\alpha}^{x x}=0
$$

which must be satisfied whenever $\frac{\partial^{\beta} u}{\partial t^{\beta}}+a u^{2} \frac{\partial^{\alpha} u}{\partial x^{\alpha}}+b \frac{\partial^{2 \alpha} u}{\partial x^{2 \alpha}}=0$. Equation (61) has the equivalent form

$$
t^{1-\beta} u_{t}+a x^{1-\alpha} u^{2} u_{x}+b(1-\alpha) x^{1-2 \alpha} u_{x}+b x^{2-2 \alpha} u_{x x}=0 .
$$

Using (11) and (12) to substitute $\eta_{\alpha}^{x}, \eta_{\alpha}^{x x}$ and $\eta_{\beta}^{t}$ into (62), replacing $u_{x x}$ by $\frac{-1}{b} t^{1-\beta} x^{2 \alpha-2} \frac{\partial u}{\partial t}-\frac{a}{b} u^{2} x^{\alpha-1} \frac{\partial u}{\partial x}-$ $(1-\alpha) x^{-1} \frac{\partial u}{\partial x}$ whenever it occurs, and equating the coefficients of the various monomials in partial derivatives of $u$, we get the full determining equations for the symmetry group of (61). Solving these equations, we obtain

$$
\tau=\frac{-4 c_{1} t}{\beta}+c_{2} t^{1-\beta}, \quad \xi=\frac{-2 c_{1}}{\alpha} x+c_{3} x^{1-\alpha}, \quad \eta=c_{1} u
$$

where $c_{1}, c_{2}$ and $c_{3}$ are arbitrary constants. Therefore, the symmetry group of (61) is spanned by the three vector fields

$$
V_{1}=t^{1-\beta} \frac{\partial}{\partial t}, \quad V_{2}=x^{1-\alpha} \frac{\partial}{\partial x}, \quad V_{3}=\frac{4 t}{\beta} \frac{\partial}{\partial t}+\frac{2 x}{\alpha} \frac{\partial}{\partial x}-u \frac{\partial}{\partial u} .
$$

The commutation relations between these vector fields are given by

$$
\left[V_{1}, V_{2}\right]=0, \quad\left[V_{1}, V_{3}\right]=4 V_{1}, \quad\left[V_{2}, V_{3}\right]=2 V_{2} .
$$

Once again the vector fields of the fractional modified Burgers equation reduce to those of the classical equations as: $\beta=\alpha=1$ [44]. tions

The one-parameter group generated by $V_{3}$ can be found by solving the corresponding characteristic equa-

$$
\frac{\alpha \mathrm{d} x}{2 x}=\frac{\beta \mathrm{d} t}{4 t}=\frac{-\mathrm{d} u}{u}
$$

and the corresponding invariants are

$$
\zeta=x t^{\frac{-\beta}{2 \alpha}}, \quad u=t^{\frac{-\beta}{4}} \Psi(\zeta) .
$$

Direct substitution of transformation (68) into equation (61) reduces (61) to a nonlinear FODE with a new independent variable. As a result, we get

$$
b \frac{d^{2 \alpha} \Psi(\zeta)}{\mathrm{d} \zeta^{2 \alpha}}+a \Psi^{2}(\zeta) \frac{d^{\alpha} \Psi(\zeta)}{\mathrm{d} \zeta^{\alpha}}-\frac{\beta}{2} \frac{\zeta^{\alpha}}{\alpha} \frac{d^{\alpha} \Psi(\zeta)}{\mathrm{d} \zeta^{\alpha}}-\frac{\beta}{4} \Psi(\zeta)=0
$$

Equation (69) can be converted by the scale $\omega=\left(\frac{\beta}{2}\right)^{\frac{1}{2 \alpha}} \zeta, \Psi(\zeta)=\left(\frac{\beta}{2}\right)^{\frac{1}{4}} \Phi(\omega)$ to the fractional equation

$$
b \frac{d^{2 \alpha} \Phi(\omega)}{\mathrm{d} w^{2 \alpha}}+\left(a \Phi^{2}(\omega)-\frac{\omega^{\alpha}}{\alpha}\right) \frac{d^{\alpha} \Phi(\omega)}{\mathrm{d} \omega^{\alpha}}-\frac{1}{2} \Phi(\omega)=0 .
$$




\section{Conclusion}

We have applied the Lie group analysis to the time-space fractional Korteweg-de Vries, modified Kortewegde Vries, Burgers, and modified Burgers equations, where the time and space derivatives are the conformable fractional derivatives. All the generating vector fields for each equation have been calculated. Thus, it is evident that the Lie group analysis can be used successfully to study conformal fractional partial differential equations. It is worth to note that the number of the generating vector fields for each of the four time-space fractional equations is the same as that of the classical equation and the generating vector fields of each of these equations reduce to that of the corresponding classical equation when $\alpha=1$ and $\beta=1$.

Using the obtained Lie symmetries, we have shown that the equations under consideration can be transformed to fractional ordinary differential equations with conformable derivative or to ordinary differential equations with classical derivative. More precisely, we have shown that the time-space fractional KdV equation can be transformed into the conformable fractional second Painlevé equation and to classical first Painlevé equation. For the time-space fractional modified KdV equation, we obtained a solution in terms of the conformable fractional second Painlevé equation. In the case of Burgers equation, we derived solutions in terms of conformable fractional Riccati and classical Riccati equations.

It should be noted that the similarity reduction method converts the time-space partial differential equation with conformable fractional derivatives to ordinary differential equations with conformable fractional derivative or with classical derivative. However, time fractional partial differential equation with conformable fractional derivative is transformed to an ordinary differential equation with classical derivative, also time fractional partial differential equation with Riemann-Liouville fractional derivative is transformed to an ordinary fractional differential equation with an Erdélyi-Kober derivative depending on a parameter $\alpha$.

It is interesting to apply the Lie group analysis to other partial differential equations with time and timespace fractional derivatives with more than two independent variables.

Open Access This article is distributed under the terms of the Creative Commons Attribution 4.0 International License (http:// creativecommons.org/licenses/by/4.0/), which permits unrestricted use, distribution, and reproduction in any medium, provided you give appropriate credit to the original author(s) and the source, provide a link to the Creative Commons license, and indicate if changes were made.

\section{References}

1. Abdeljawad, T.: On conformable fractional calculus. J. Comput. Appl. Math. 279, 57-66 (2015)

2. Abdeljawad, T.; Al Horani, M.; Khalil, R.: Conformable fractional semigroups of operators. J. Semigroup Theory Appl. 2015, 7 (2015)

3. Anderson, D.R.; Ulness, D.J.: Results for conformable differential equations. (preprint) (2016)

4. Atangana, A.; Baleanu, D.; Alsaedi, A.: New properties of conformable derivative. Open Math. 13, 889-898 (2015)

5. Bakkyaraj, T.; Sahadevan, R.: Invariant analysis of nonlinear fractional ordinary differential equations with RiemannLiouville fractional derivative. Nonlinear Dyn. 80(1-2), 447-455 (2015)

6. Benkhettou, N.; Hassani, S.; Torres, D.F.: A conformable fractional calculus on arbitrary time scales. J. King Saud Univ. Sci. 28(1), 93-98 (2016)

7. Bluman, G.W.; Kumei, S.: Symmetries and Differential Equations. Vol. 81. Springer, New York (1989)

8. Bluman, G.W.; Anco, S.C.: Symmetry and Integration Methods for Differential Equations. Springer, Heidelberg (2002)

9. Bluman, G.W.; Cheviakov, A.F.; Anco, S.C.: Applications of Symmetry Methods to Partial Differential Equations. Springer, New York (2010)

10. Buckwar, E.; Luchko, Y.: Invariance of a partial differential equation of fractional order under the Lie group of scaling transformations. J. Math. Anal. Appl. 227, 81-97 (1998)

11. Caputo, M.: Linear model of dissipation whose $Q$ is almost frequency dependent II. Geophys. J. R. Ast. Soc. 13, 529-539 (1967)

12. Diethelm, K.: The Analysis of Fractional Differential Equations. Springer, New York (2010)

13. Djordjevic, V.D.; Ttanackovic, T.M.: Similarity solution to nonlinear heat conduction and Burgers/Korteweg-de Vries fractional equation. J. Comput. Appl. Math. 222, 701-714 (2008)

14. Freire, I.L.: Note on Lie point symmetries of Burgers equations. Trends Appl. Comput. Math. 11(2), 151-157 (2010)

15. Fuat, U.; Mehmet, Z.S.: Explicit bounds on certain integral inequalities via conformable fractional calculus. Cogent Math. 4, 1277505 (2017)

16. Gandarias, M.L.; Khalique, C.M.: Symmetries, solutions and conservation laws of a class of nonlinear dispersive wave equations. Commun. Nonlinear Sci. Numer. Simul. 32, 114-121 (2016)

17. Gazizov, R.K.; Kasatkin, A.A.; Lukashchuk, SYu: Continuous transformation groups of fractional differential equations. Vestnik USATU 9, 125-135 (2007) 
18. Gazizov, R.K.; Kasatkin, A.A.; Lukashchuk, SYu: Symmetry properties of fractional diffusion equations. Phys. Scr. T136, 014-016 (2009)

19. Hammad, M.A.; Khalil, R.: Abel's formula and Wronskian for conformable fractional differential equations. Int. J. Differ. Equ. Appl. 13, 177-183 (2014)

20. Hashemi, M.S.: Group analysis and exact solutions of the time fractional Fokker-Planck equation. Phys. A Stat. Mech. Appl. 417, 141-149 (2015)

21. Hu, J.; Ye, Y.J.; Shen, S.F.; Zhang, J.: Lie symmetry analysis of the time-fractional KdV-type equation. Appl. Math. Comput. 233, 439-444 (2014)

22. Huang, Q.; Zhdanov, R.: Symmetries and exact solutions of the time-fractional Harry-Dym equation with Riemann-Liouville derivative. Physica A 409, 110-118 (2014)

23. Ibragimov, N.H.: Handbook of Lie Group Analysis of Differential Equations, vol. 1. CRC Press, Boca Raton (1994)

24. Iyiola, O.S.; Nwaeze, E.R.: Some new results on the new conformable fractional calculus with application using D'Alambert approach. Prog. Fract. Differ. Appl. 2, 115-122 (2016)

25. Jafari, H.; Kadkhoda, N.; Baleanu, D.: Fractional Lie group method of the time-fractional Boussinesq equation. Nonlinear Dyn. 81(3), 1569-1574 (2015)

26. Jumarie, G.: Modified Riemann-Liouville derivative and fractional Taylor series of non-differentiable functions further results. Comput. Math. Appl. 51, 1367-1376 (2006)

27. Katugampola, U.: A new fractional derivative with classical properties, arXiv: 1410.6535v2 (2014)

28. Khalil, R.; Al Horani, M.; Yousef, A.; Sababheh, M.: A new definition of fractional derivative. J. Comput. Appl. Math. 264, 65-70 (2014)

29. Kiryakova, V.: Pitman Research Notes in Mathematics, vol. 301. Longman, London (1994)

30. Lukashchuk, SYu; Makunin, A.V.: Group classfication of nonlinear time-fractional diffusion equation with a source term. Appl. Math. Comput. 257, 335-343 (2015)

31. Machado, J.T.; Kiryakova, V.; Mainardi, F.: Recent history of fractional calculus. Commun. Nonlinear Sci. Numer. Simul. 16(3), 1140-1153 (2011) (2011)

32. Michal, P., Škripková, L.P.: Sturm's theorems for conformable fractional differential equations. Math. Commun. 21, 273-281 (2016)

33. Miller, K.S.; Ross, B.: An Introduction to the Fractional Calculus and Fractional Differential Equations. Wiley, New York (1993)

34. Oldham, K.B.; Spanier, J.: The Fractional Calculus. Academic Press, London (1974)

35. Olver, P.J.: Applications of Lie Groups to Differential Equations, GTM 107, 2nd edn. Springer, Berlin (1993)

36. Ouhadan, A.; Elkinani, E.H.: Exact Solutions of Time-Fractional Kolmogorov Equation by Using Lie Symmetry Analysis. J. Fract. Calc. Appl. 5, 97-104 (2014)

37. Podlubny, I.: Fractional Differential Equations. Academic Press, San Diego (2006)

38. Rui, W.; Zhang, X.: Lie symmetries and conservation laws for the time fractional Derrida-Lebowitz-Speer-Spohn equation. Commun. Nonlinear Sci. Numer. Simul. 34, 38-44 (2016)

39. Sahadevan, R.; Bakkyaraj, T.: Invariant analysis of time-fractional generalized Burgers and Korteweg-de Vries equations. J. Math. Anal. Appl. 393, 341-347 (2012)

40. Samko, S.G.; Kilbas, A.A.; Marichev, O.L.: Fractional Integrals and Derivatives: Theory and Applications. Gordon and Breach, New York (1993)

41. Sarikaya, M.Z.: Gronwall type inequality for conformable fractional integrals. RGMIA Res. Rep. Collect. 19, 122 (2016)

42. Tayyan, B.A.; Sakka, A.H.: Symmetries and Exact Solutions of Conformable Fractional Partial Differential Equations. Palestine J. Math. (to appear)

43. Usta, F.; Sarikaya, M.Z.: On generalization conformable fractional integral inequalities. RGMIA Res. Rep. Collect. 19(123) 8 (2016)

44. Vaneeva, O.O.; Sophocleous, C.; Leach, P.G.L.: Lie symmetries of generalized Burgers equations: application to boundaryvalue problems. J. Eng. Math. 91(1), 165-176 (2015)

45. Wang, G.; Xu, T.: Symmetry properties and explicit solutions of the nonlinear time fractional KdV equation. Bound. Value Probl. 2013, 232 (2013)

46. Wang, G.; Xu, T.: Invariant analysis and exact solutions of nonlinear time-fractional Sharma-Tasso-Olver equation by Lie group analysis. Nonlinear Dyn. 76, 571-80 (2014)

47. Wang, G.; Liu, X.; Zhang, Y.: Lie symmetry analysis to the time-fractional generalized fifth-order KdV equation. Commun. Nonlinear Sci. Numer. Simul. 18, 2321-2326 (2013)

48. Wang, G.; Kara, A.H.; Fakhar, K.: Symmetry analysis and conservation laws for the class of time-fractional nonlinear dispersive equation. Nonlinear Dyn. 82, 281-287 (2015)

49. Wu, G.C.: A fractional Lie group method for anomalous diffusion equations. Commun. Frac. Calc. 1, 27-31 (2010)

50. Zhao, D.; Luo, M.: General conformable fractional derivative and its physical interpretation. Calcolo 54, 1-15 (2017)

51. Zheng, A.; Feng, Y.; Wang, W.: The Hyers-Ulam stability of the conformable fractional differential equation. Math. Aeterna 5, 485-492 (2015)

Publisher's Note Springer Nature remains neutral with regard to jurisdictional claims in published maps and institutional affiliations. 